\title{
Stochastic Resonance in an Extended FitzHugh-Nagumo System: the Role of Selective Coupling
}

\author{
Claudio J. Tessone, ${ }^{\mathrm{a}}$ Horacio S. Wio ${ }^{\mathrm{b}, *}$ \\ ${ }^{a}$ Institut Mediterrani d'Estudis Avançats (IMEDEA-CSIC), Universitat de les Illes \\ Balears, E07122 Palma de Mallorca, Spain. \\ b Instituto de Física de Cantabria, Universidad de Cantabria and CSIC, E39005 \\ Santander, Spain.
}

\begin{abstract}
Here we present a study of stochastic resonance in an extended FitzHugh-Nagumo system with a field dependent activator diffusion. We show that the system response (here measured through the output signal-to-noise ratio) is enhanced due to the particular form of the non-homogeneous coupling. Such a result supports previous ones obtained in a simpler scalar reaction-diffusion system and shows that such an enhancement, induced by the field dependent diffusion -or selective coupling-, is a robust phenomenon.
\end{abstract}

Key words: Stochastic resonance, Spatially-extended systems, Field dependent diffusion

PACS: 05.45.-a, 05.40.Ca

\section{Introduction}

Stochastic resonance (SR) is one of the most interesting noise-induced phenomena that arises from the interplay between deterministic and random dynamics in a nonlinear system (1). A large number of examples showing SR occur in extended systems: for example, diverse experiments were carried out

* Corresponding Author: H.S. Wio, Instituto de Física de Cantabria, 39005 Santander, Spain

Email addresses: tessonec@imedea.uib.es (Claudio J. Tessone,), wio@ifca.unican.es (Horacio S. Wio).

Preprint submitted to Elsevier Science 30 August 2018 
to explore the role of SR in sensory and other biological functions (2) or in chemical systems (3). These, together with the possible technological applications, motivated many recent studies showing the possibility of achieving an enhancement of the system response by means of the coupling of several units in what conforms an extended medium $(4 ;$; $; 6)$.

In previous works $(5 ;$; $)$ we have studied the stochastic resonant phenomenon in extended systems, when transitions between two different spatial patterns occurs, exploiting the concept of the non-equilibrium potential (NEP) (7); 8): a Lyapunov functional of the associated deterministic system that, for nonequilibrium systems, plays a role similar to that of a thermodynamic potential in equilibrium thermodynamics. Such NEP characterizes the global properties of the dynamics: attractors, relative (or nonlinear) stability of these attractors, height of the barriers separating attraction basins and, in addition, allowing us to evaluate the transition rates among the different attractors. In another work (9) we have also shown that, for a scalar reaction-diffusion system with a density-dependent diffusion and a known form of the NEP, the non-homogeneous spatial coupling changes the effective dynamics of the system and contributes to enhance the SR phenomenon.

Here we report on a study of SR in an extended system: an array of FitzHughNagumo (11) units, with a density-dependent (diffusive-like) coupling. The NEP for this system was found within the excitable regime and for particular values of the coupling strength (6). In the general case, however, the form of the NEP has not been found yet. Nevertheless, the idea of the existence of such a NEP is always underlying our study. Hence, we have resorted to an study based on numerical simulations, analyzing the influence of different parameters on the system response. The results show that the enhancement of the signal-to-noise ratio found for a scalar system (9) is robust, and that the indicated non-homogeneous coupling could clearly contribute to enhance the SR phenomenon in more general situations.

\section{Theoretical Framework}

\subsection{The Model}

For the sake of concreteness, we consider a simplified version of the FitzHughNagumo (6; 8; 11) model. This model has been useful for gaining qualitative insight into the excitable and oscillatory dynamics in neural and chemical systems (10). It consist of two variables, in one hand $u$, a (fast) activator field that in the case of neural systems represents the voltage variable, while in chemical systems represents a concentration of a self-catalytic species. On 


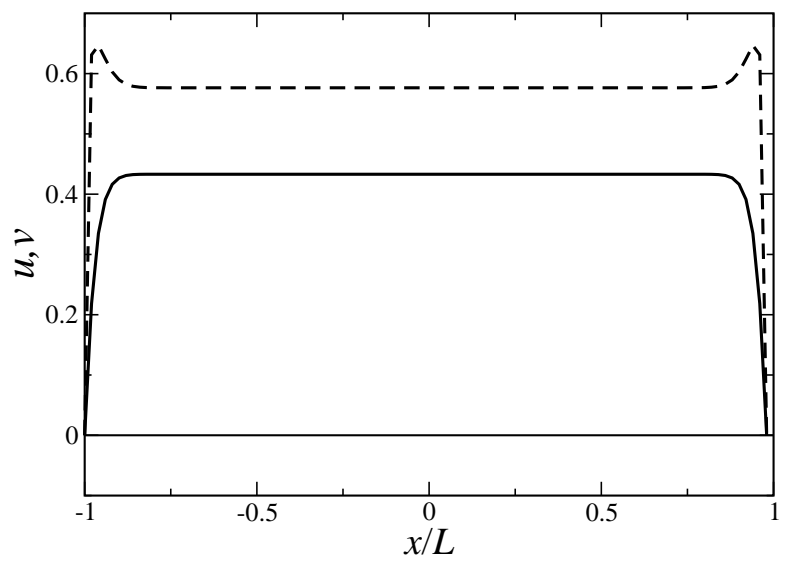

Fig. 1. We show the stable patterns that arise in the system. There is one stable pattern that is identically zero, i.e. $P_{0}^{u}(x)=P_{0}^{v}(x)=0$ and another which is non-zero $\left(P_{1}^{u}(x), P_{1}^{v}(x)\right)$. The patterns for the fields $u(x)$ and $v(x)$ are plotted in dashed and solid lines, respectively. The parameters, are $D_{u}=0.3, D_{v}=1, h=2$.

the other hand $v$, the inhibitor field, associated with the concentration of potassium ions in the medium (within a neural context), that inhibits the generation of the $u$ species (in a chemical reaction). Instead of considering the usual cubic like nonlinear form, we use a piece-wise linear version

$$
\begin{aligned}
\epsilon \frac{\partial u(x, t)}{\partial t} & =\frac{\partial}{\partial x}\left(D_{u}(u) \frac{\partial u}{\partial x}\right)+f(u)-v+\xi(x, t) \\
\frac{\partial v(x, t)}{\partial t} & =\frac{\partial}{\partial x}\left(D_{v}(v) \frac{\partial u}{\partial x}\right)+\beta u-\alpha v
\end{aligned}
$$

where $f(u)=-u+\Theta\left(u-\phi_{c}\right)$, and $\xi(x, t)$ is a $\delta$-correlated white Gaussian noise, that is $\langle\xi(x, t)\rangle=0$ and $\left\langle\xi(x, t) \xi\left(x^{\prime}, t^{\prime}\right)\right\rangle=2 \gamma \delta\left(x-x^{\prime}\right) \delta\left(t-t^{\prime}\right)$. Here $\gamma$ indicates the noise intensity and $\phi_{c}$ is the "discontinuity" point, at which the piece-wise linearized function $f(u)$ presents a jump. In what follows, the parameters $\alpha$ and $\beta$ are fixed as $\alpha=0.3$ and $\beta=0.4$. Finally, $\epsilon$ is the parameter that indicates the time-scale ratio between activator and inhibitor variables, and is set as $\epsilon=0.03$. We consider Dirichlet boundary conditions at $x= \pm L$. Although the results are qualitatively the same as those that could appear considering the usual FitzHugh-Nagumo equations, this simplified version allows us to compare directly with the previous analytical results for this system (6).

As in (9), we assume that the diffusion coefficient $D_{u}(u)$ is not constant, but depends on the field $u$ according to $D_{u}(u)=D_{u}\left[1+h \Theta\left(u-\phi_{c}\right)\right]$. This form implies that the value of $D_{u}(u)$ depends "selectively" on whether the field $u$ fulfills $u>\phi_{c}$ or $u<\phi_{c}$. $D_{u}$ is the value of the diffusion constant without such 
"selective" term, and $h$ indicates the size of the difference between the diffusion constants in both regions (clearly, if $h=0$ then $D_{u}(u)=D_{u}$ constant). $D_{v}(v)$ is the diffusion for the inhibitor $v$, that here we assume to be homogeneously constant.

It is worthwhile noting that when the parameter $h$ is large enough, under some circumstances the coupling term might become negative. This is what is known as "inhibitory coupling" (12). This is a very interesting kind of coupling that has attracted much attention in the last years, both in neural and chemical context, that we will not discuss here.

This system is known to exhibit two stable stationary patterns. One of them is $u(x)=0, v(x)=0$, while the other is one with non-zero values and can be seen in Fig. 1. We will denote with $P_{0}^{u, v}(x)$ and $P_{1}^{u, v}(x)$, the patterns for $u$ and $v$ fields. Further, we consider that an external, periodic, signal enters into the system through the value of the threshold $\phi_{c}$,

$$
\phi_{c}(t)=\phi_{c}+\delta \phi \cos (\omega t)
$$

where $\omega$ is the signal frequency, and $\delta \phi$ its intensity.

All the results shown in this paper were obtained through numerical simulations of the system. The second order spatially discrete version of the system indicated in Eqs. $(1,2)$ reads

$$
\begin{array}{ll}
\dot{u}_{i}=D_{u, i}\left(u_{i-1}+u_{i+1}-2 u_{i}\right)+\left(D_{u, i+1}-D_{u, i-1}\right)\left(u_{i+1}+u_{i-1}\right) \\
\dot{v}_{i}=D_{v}\left(v_{i-1}+v_{i+1}-2 v_{i}\right)+\beta u_{i}-\alpha v_{i} & +f\left(u_{i}\right)-v_{i}+\xi_{i}(t)
\end{array}
$$

We have performed extensive numerical simulations of this set of equations exploiting the Heun's algorithm (13).

\subsection{Response's Measures}

Since the discovery of the stochastic resonance phenomenon, several different forms of characterizing it have been introduced in the literature. Some examples are: (i) output signal-to-noise ratio (SNR) (1); 14), (ii) the spectral amplification factor (SAF) (15; 16), (iii) the residence time distribution (17; 18), and, more recently, (iv) information theory based tools (19; 20; 21). Along this paper, we will use the output SNR at the driving frequency $\omega$.

In this spatially-extended system, there are different ways of measuring the overall system response to the external signal. In particular, we evaluated the 
output SNR in three different ways (the units being given in $\mathrm{dB}$ )

- SNR for the element $N / 4$ of the chain evaluated over the dynamical evolution of $u_{N / 4}$, that we call $S N R_{1}$.

- SNR for the middle element of the chain evaluated over the dynamical evolution of $u_{N / 2}$, that we call $S N R_{2}$. Having Dirichlet boundary conditions, the local response depends on the distance to the boundaries.

- In order to measure the overall response of the system to the external signal, we computed the SNR as follows: We digitized the system dynamics to a dichotomic process $s(t)$ : At time $t$ the system has an associated value of $s(t)=1(0)$ if the Hilbert distance to pattern $1(0)$ is lower than to the other pattern. Stated in mathematical terms, we computed the distance $\mathcal{D}_{2}[\cdot, \cdot]$ defined by

$$
\mathcal{D}_{2}[f, g]=\left(\int_{-L}^{L} d x(f(x)-g(x))^{2}\right)^{1 / 2}
$$

in the Hilbert space of the real-valued functions in the interval $[-L, L]$, i.e. $\mathcal{L}_{2}$. At time $t$, a digitized process is computed by means of

$$
s(t)=\left\{\begin{array}{ll}
1 & \text { if } \mathcal{D}_{2}\left[P_{1}^{u}(x), u(x, t)\right]<\mathcal{D}_{2}\left[P_{0}^{u}(x), u(x, t)\right] \\
0 & \text { if } \mathcal{D}_{2}\left[P_{1}^{u}(x), u(x, t)\right] \geq \mathcal{D}_{2}\left[P_{0}^{u}(x), u(x, t)\right]
\end{array},\right.
$$

We call this measure $S N R_{p}$.

\section{Results}

As indicated above, Eqs. (4) and (5) have been integrated by means of the Heun method (22). We have fixed the parameters $\epsilon=0.03, \phi_{c}=0.52$ and adopted an integration step of $\Delta t=10^{-3}$. For the signal frequency we adopted $\omega=2 \pi / 3.2=1.9634295 \ldots$. The simulation was repeated 250 times for each parameter set, and the SNR was computed by recourse of the average power spectral density.

Figure 2 depicts the results for the different SNR's measures we have previously defined as function of the noise intensity $\gamma$. We adopted the following values: $\delta \phi=0.4, D_{v}=1$. and $N=51$. In all three cases it is apparent that there is an enhancement of the response for $h>0$, when compared with the $h=0$ case, while for $h<0$ the response is smaller.

In Fig. 3 we show the same three response's measures, but now as a function of $h$. We have plotted the maximum of each SNR curve, for three different 

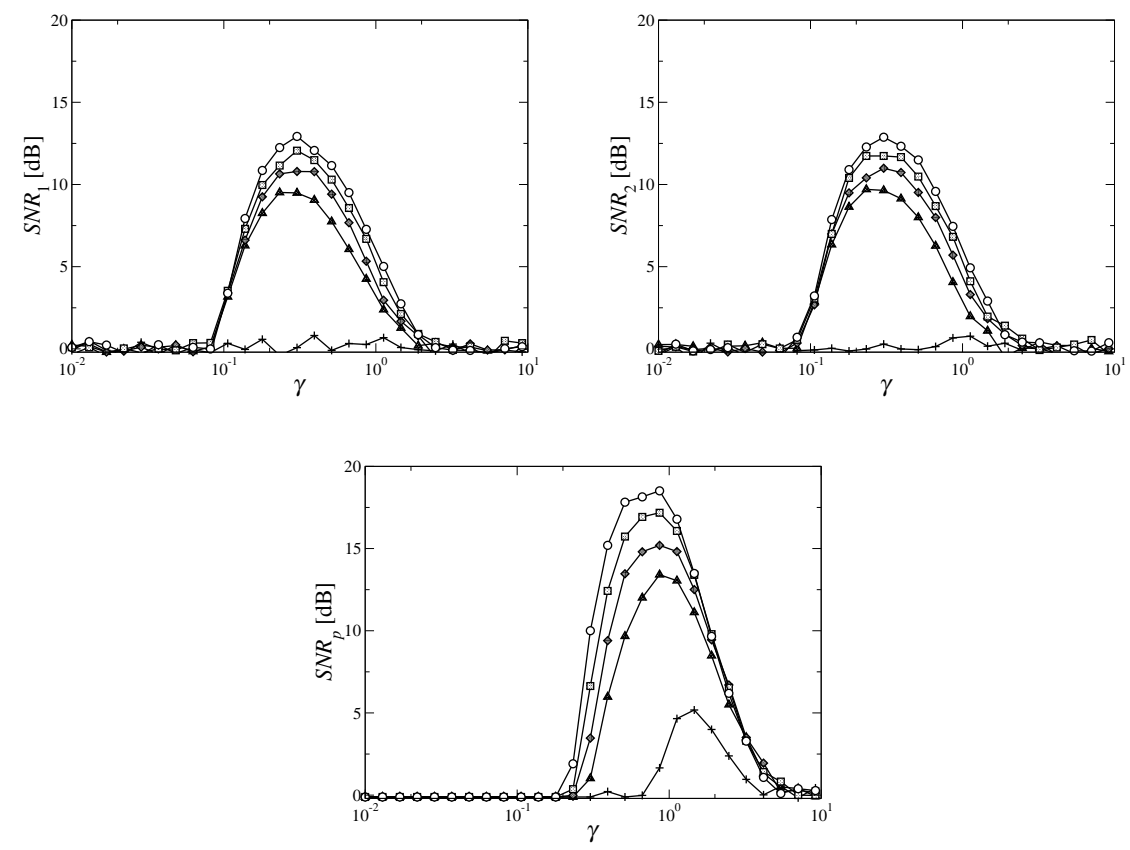

Fig. 2. SNR vs. $\gamma$, the noise intensity, for the three different measures we use. The parameters are $\delta \phi=0.4, D_{v}=1 ., \omega=2 \pi / 3.2, N=51$. The different curves represent different values of $h$, showing an enhancement of the response to the external signal for $\gamma>0$. In particular it is shown: $h=-2(+), h=-1(\triangle), h=0$ $(\diamond), h=1(\square)$ and $h=2(\bigcirc)$.

values of the noise intensity, and for $\delta \phi=0.4, D_{v}=1 ., \gamma=0.01,0.1,0.3$, $D_{u}=0.3$, and $N=51$. It is clear that there exists an optimal value of $\gamma$ such that, for such a value, the phenomenon is stronger (that is, the response is larger). It is apparent the rapid fall in the response for $h<0$.

In figure 4 we show the dependance of SNR on $h$, for different values of the diffusion which depends on the activator density $D_{u}$. It is apparent that the response becomes larger when the value of $D_{u}$ is larger. However, as was discussed in $(5 ; 6)$, it is clear that for still larger values of $D_{u}$, the symmetry of the underlying potential (that is the relative stability between the attractors) is broken and the response finally falls-down.

Figure 5 shows the results of the SNR, but now as function of $D_{u}$, the activator diffusivity, for different values of $\gamma$, and for $\delta \phi=0.4, D_{v}=1$. and $N=51$. It can be seen that, independently from the coupling strength $D_{u}$, the response to the external signal grows with the selectiveness of the coupling, showing the robustness of the phenomenon.

Next, in figure 6 , we present the results for the SNR as function of $D_{v}$, the activator diffusivity, for different values of $\gamma$, and for $\delta \phi=0.4, D_{u}=0.3$, and $N=51$. We see that for $h \geq 0$ the response is more or less flat, however, it is again apparent the SNR's enhancement for $h>0$. For $h<0$ we see that 

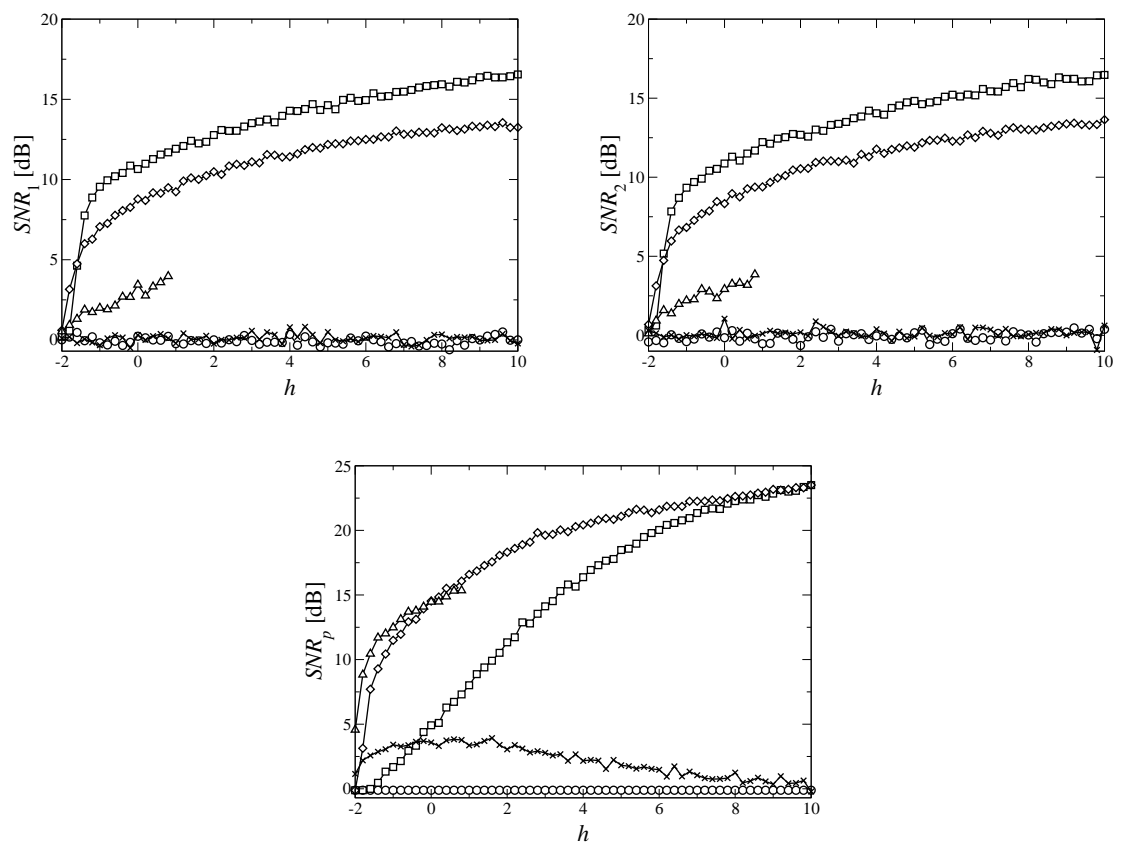

Fig. 3. SNR vs. $h$, the selectiveness of coupling, for the three different measures we use. The parameters are $\delta \phi=0.4, D_{v}=1$., $\gamma=0.032(\bigcirc), 0.32(\square), 0.6(\diamond), 1.2(\square), 3.2(\times), D_{u}=0.3, N=51$.
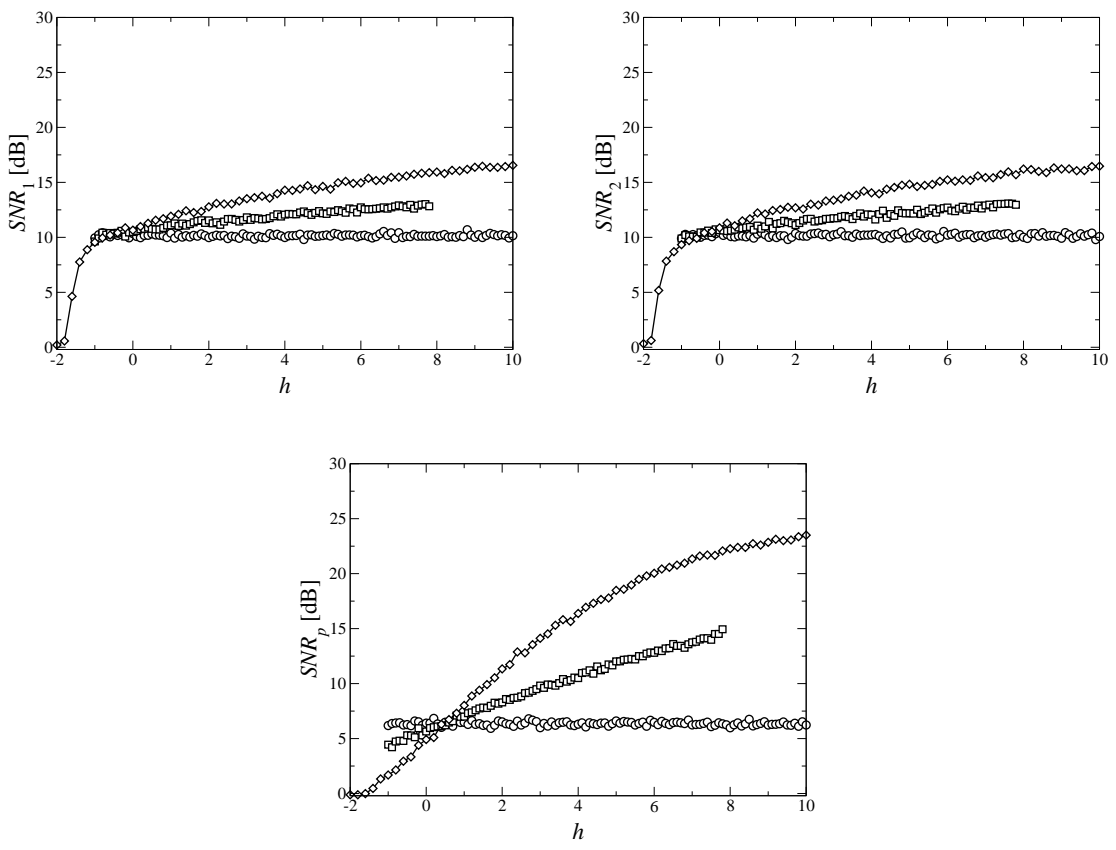

Fig. 4. SNR vs. $h$, the selectiveness of coupling, for different values of $D_{u}$. The parameters are $\delta \phi=0.4, D_{v}=1 ., \gamma=0.32, D_{u}=0.0(\bigcirc), 0.1(\square), 0.3(\diamond), N=51$. 

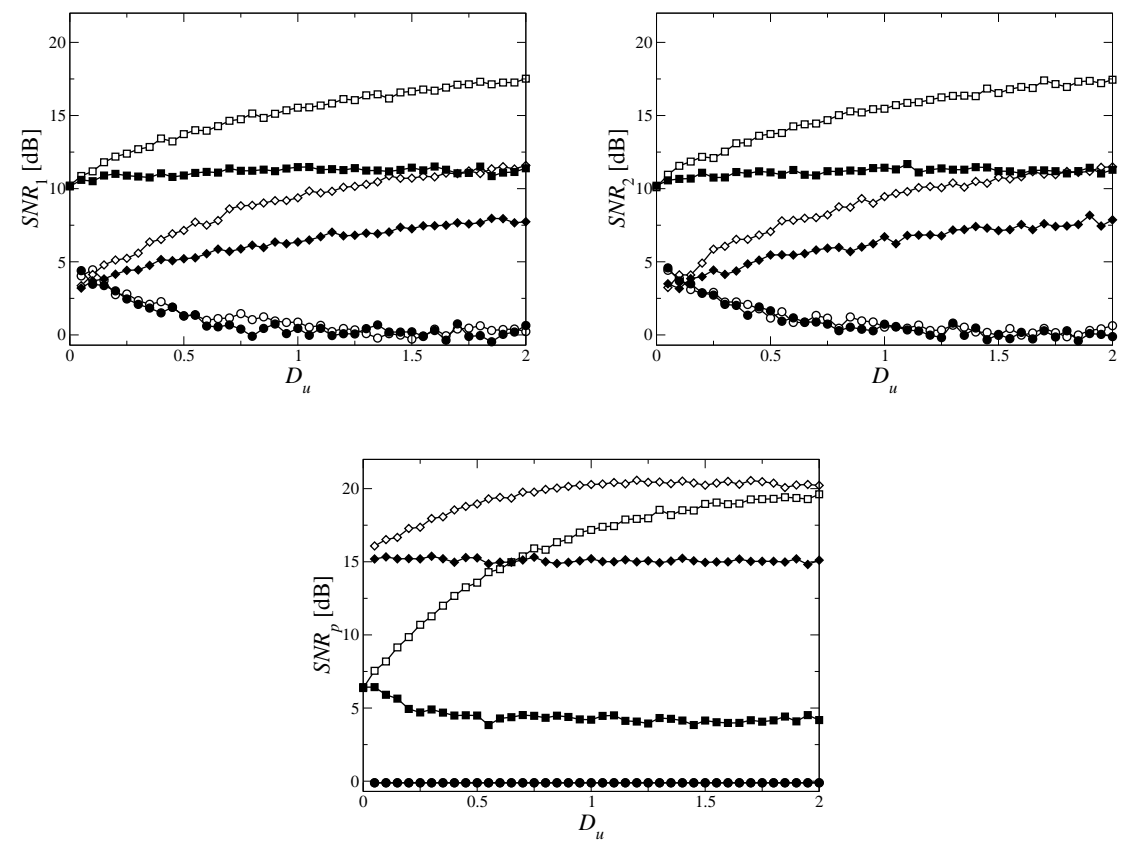

Fig. 5. SNR vs. $D_{u}$, the diffusiveness in activator variable $u$, for the three different measures we use. The parameters are $\delta \phi=0.4, D_{v}=1$., $\gamma=0.1(\bigcirc), 0.32(\square), 1.0(\diamond)$, while the white symbols represent $h=2$ and the black ones, $h=0$. The system size is $N=51$.

the system's response decays very fast with increasing $D_{v}$. This effect could be associated to the fact (as found in those cases where the NEP is known $(5 ;$; 6$)$ ) that in the underlying NEP the bistability is lost as a consequence of the disappearance of some of the attractors (8).

Finally, in figure 7 we depict the same three SNR's measures but as a function of $N$, the system size. For the two measures $S N R_{1}$ and $S N R_{2}$, we see that, for different values of $h$ and $\gamma$, the response is very flat, and do not seems to be too much dependent on $N$. It is clear that there is an increase of the response when $h$ increases. At variance, for $S N R_{p}$, the dependence to $N$ is apparent: the SNR decays to zero, in a fast or slow way, depending of $h=0$ or $h>0$. Here $\delta \phi=0.4, D_{u}=0.3, D_{v}=1 ., \gamma=0.01,0.1,0.3$.

\section{Conclusions}

We have analyzed a simplified version of the FitzHugh-Nagumo model (ㅎ; ; ; 11), where the activator's diffusion is density-dependent. Such a system, when both diffusions are constant (that is: $D_{u}>0$ and $D_{v}=0$ ), has a known form of the NEP (6) $)$. However, in the general case we have not been able to find the form of the NEP (but the idea of such a NEP is always underlying our 

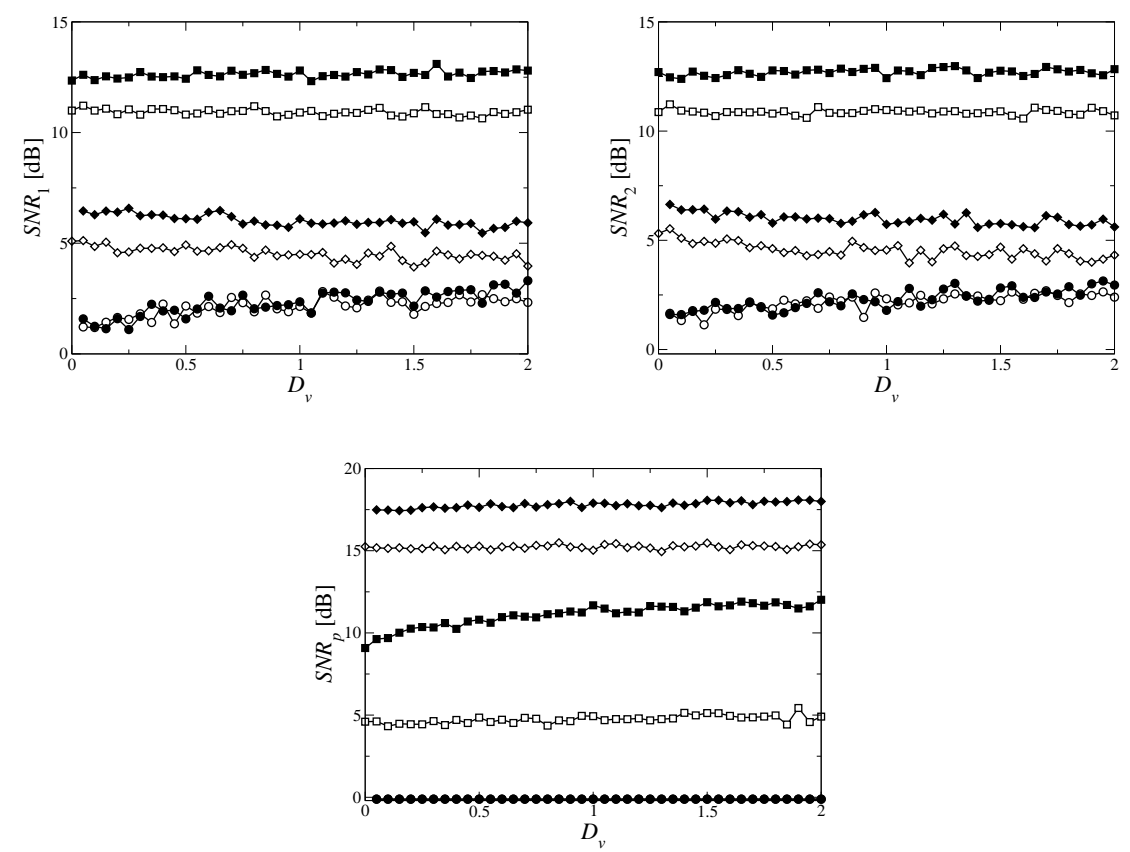

Fig. 6. SNR vs. $D_{v}$, the diffusiveness in the inhibitor variable $v$. The parameters are $\delta \phi=0.4, D_{u}=0.3, \omega=2 \pi / 3.2, N=51 . \gamma=0.1(\bigcirc), 0.32(\square), 1.0(\diamond)$, while the white symbols represent $h=2$ and the black ones, $h=0$.
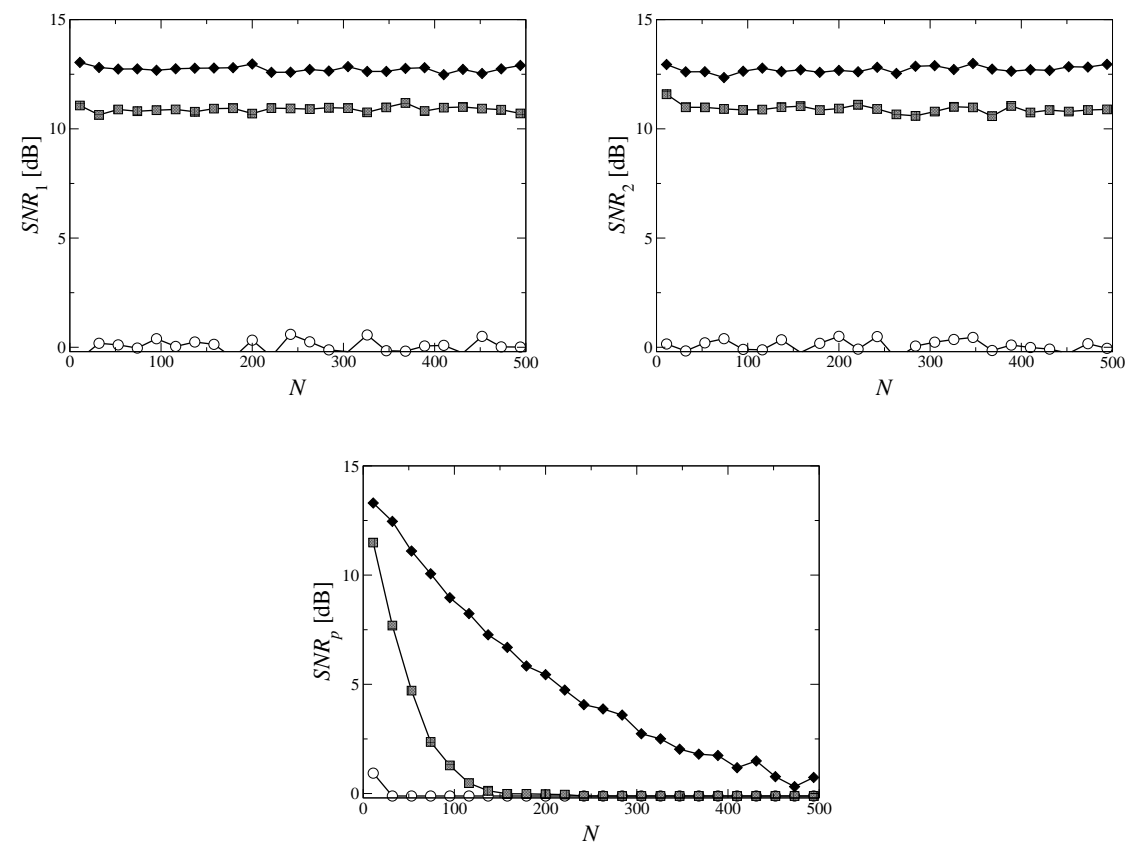

Fig. 7. SNR vs. $N$, the system size, for the three different measures we use. The parameters are $\delta \phi=0.4, D_{u}=0.3, D_{v}=1 ., \gamma=0.32$, $h=-2(\bigcirc), 0$ (grey squares), $2(\diamond)$. 
analysis) and we have to resort to an analysis based on numerical simulations.

Through the numerical approach we have studied the influence of the different parameters on the system response. From the results it is apparent the enhancement of the output SNR as $h$, the selectivity parameter, is increased. This is seen through three different ways of characterizing the system's response. We can conclude that the phenomenon of enhancement of the SNR, due to a selectivity in the coupling, initially found for a scalar system (9) is robust, and that the indicated nonhomogeneous coupling could clearly contribute to enhance the SR phenomenon in very general systems. This phenomenon is also robust to variations of the parameter that controls the selectiveness of the coupling, up to a point that even in the case of inhibitory coupling the phenomenon holds.

An aspect worth to be studied in detail is the dependence of the SNR on $N$, the number of coupled units. In this way we could analyze the dependence of the so called system size stochastic resonance $(23 ; 24)$ on the selective coupling. The thorough study of this problem will be the subject of further work.

Acknowledgments: HSW thanks to the European Commission for the award of a Marie Curie Chair at the Universidad de Cantabria, Spain.

\section{References}

[1] L. Gammaitoni, P. Hänggi, P. Jung, F. Marchesoni, Rev. Mod. Phys. 70, 223 (1998).

[2] J.K. Douglas et al., Nature 365, 337 (1993);

J.J. Collins et al., Nature 376, 236 (1995);

S.M. Bezrukov, I. Vodyanoy, Nature 378, 362 (1995).

[3] A. Guderian, et al; J. Phys. Chem. 100, 4437 (1996);

A. Förster, et al, J. Phys. Chem. 100, 4442 (1996);

W. Hohmann, et al, J. Phys. Chem. 100, 5388 (1996).

[4] J.F. Lindner et al., Phys. Rev. E 53, 2081 (1996);

J.F. Lindner et al., Phys. Rev. Lett. 75, 3 (1995).

[5] H.S. Wio, Phys. Rev. E 54, R3045 (1996);

F. Castelpoggi, H.S. Wio, Europhys. Lett. 38, 91 (1997);

ibidem, Phys. Rev. E 57, 5112 (1998);

S. Bouzat, H.S. Wio, Phys. Rev. E 59, 5142 (1999).

[6] H.S. Wio, S. Bouzat, B. von Haeften, in Proc. 21 ${ }^{\text {st }}$ IUPAP International Conference on Statistical Physics, STATPHYS21, A.Robledo, M. Barbosa (Eds.), Physica A 306C 140-156 (2002).

[7] R.Graham, in Instabilities and Nonequilibrium Structures, Eds. E. Tirapegui and D. Villaroel (D. Reidel, Dordrecht, 1987); 
R. Graham, T. Tel, Phys. Rev. A 42, 4661 (1990);

R. Graham, T. Tel, in Instabilities and Non-equilibrium Structures III, E. Tirapegui, W. Zeller, eds. (Kluwert, 1991);

H.S. Wio, in 4th. Granada Seminar in Computational Physics, Eds. P. Garrido, J. Marro (Springer-Verlag, Berlin, 1997), pg.135.

[8] G. Izús et al, Phys. Rev. E 52, 129 (1995);

G. Izús et al, Int. J. Mod. Physics B 10, 1273 (1996);

D.H. Zanette, H.S. Wio, R. Deza, Phys. Rev. E 53, 353 (1996);

F. Castelpoggi, H.S. Wio, D.H. Zanette, Int. J. Mod. Phys. B 11, 1717 (1997);

G. Drazer, H.S. Wio, Physica A 240, 571 (1997).

[9] B. von Haeften, R. Deza, H.S. Wio, Phys. Rev. Lett. 84, 404 (2000).

[10] B. Lindner, J. García-Ojalvo, A. Neiman, L. Schimansky-Geier, Phys. Rep. 392, 321 (2004).

[11] H.S. Wio, An Introduction to Stochastic Processes and Nonequilibrium Statistical Physics (World Scientific, 1994);

A.S. Mikhailov, Foundations of Synergetics I, (Springer-Verlag, 1990).

[12] P. Dayan and L. F. Abbott, Theoretical neuroscience: computational and mathematical modeling of neural systems, Computational neuroscience (MIT Press, Cambridge, 2001).

[13] J. García-Ojalvo and J.M. Sancho, Noise in Spatially Extended Systems (Springer, New York, 1999).

[14] B. McNamara, K. Wiesenfeld, Phys. Rev. A 39, 4854 (1989).

[15] P. Jung and P. Hänggi, Europhys. Lett. 8, 505 (1989);

[16] P. Jung, P. Hänggi, Phys. Rev. A 44, 8032 (1991).

[17] I. Goychuk and P. Hänggi, Phys. Rev. E 61, 4272 (2000).

[18] L. Gammaitoni, F. Marchesoni, E. Menichella-Saetta, S. Santuci; Phys.

Rev. Lett. 62, 39 (1989).

[19] A. Bulsara, A. Zador, Phys. Rev. E 54, R2185 (1996).

[20] L. Schimansky-Geier, J.A. Freund, A.B. Neiman, B. Shulgin, Int. J. of Bif. \& Chaos 8, 869 (1997).

[21] C.J. Tessone, A. Plastino, H.S. Wio, Physica A 326, 37 (2003).

[22] M. San Miguel, R. Toral, Stochastic Effects in Physical Systems, in Instabilities and Nonequilibrium Structures VI, E. Tirapegui, W. Zeller, Eds.

(Kluwer Ac.Press, 1999).

[23] G. Schmid, I. Goychuk, and P. Hänggi, Europhys. Lett. 56, 22 (2001);

P.Jung and J.W. Shuai, Europhys. Lett. 56, 29 (2001).

[24] G. Schmid, I. Goychuk, P. Hänggi, Europhys. Lett. 56, 22 (2001);

A. Pikovsky, A. Zaikin, M.A. de la Casa, Phys. Rev. Lett. 88, 050601 (2002);

R. Toral, C. Mirasso, J. Gunton, Europhys. Lett. 61, 162 (2003);

B. von Haeften, G.G. Izús and H.S.Wio, Phys. Rev. E 72, 021101 (2005). 